\title{
Isostructural Phase Transition in Bismuth Oxide Chloride induced by Redistribution of Charge under High Pressure
}

Jinggeng Zhao, ${ }^{*}{ }^{\dagger}$ Lingling Xu,${ }^{\star}$ Yang Liu,${ }^{\star}$ Zhenhai $\mathrm{Yu},{ }^{\S}$ Chunyu Li,${ }^{\S}$ Yi Wang,,${ }^{*}{ }^{\dagger}$ Zhiguo Liu* ${ }^{*}$

${ }^{\dagger}$ Natural Science Research Center, Academy of Fundamental and Interdisciplinary Sciences,

Harbin Institute of Technology, Harbin 150080, China

${ }^{*}$ Key Laboratory of Photonic and Electric Bandgap Materials, Ministry of Education, School of Physics and Electronic Engineering, Harbin Normal University, Harbin 150025, China

${ }^{\S}$ Center for High Pressure Science and Technology Advanced Research, Shanghai, 201203, China

${ }^{\#}$ Department of Physics, Harbin Institute of Technology, Harbin 150080, China

E-mail: zhaojinggeng@163.com (J.G.Z.), yw@ hit.edu.cn (Y.W.), liuzhiguo@hit.edu.cn (Z.G.L.). 
Table S1. The atomic coordination parameters of BiOCl under high pressure.

\begin{tabular}{|c|c|c|c|c|c|c|c|}
\hline$P(\mathbf{G P a})$ & $a(\AA)$ & $c(\AA)$ & $z_{\mathrm{Bi}}$ & $z_{\mathrm{Cl}}$ & $\boldsymbol{R}_{\mathrm{wp}}$ & $R_{\mathrm{p}}$ & $\chi^{2}$ \\
\hline $0.49(1)$ & $3.8841(1)$ & $7.3409(5)$ & $0.1758(2)$ & $0.6436(11)$ & $7.27 \%$ & $4.87 \%$ & 1.990 \\
\hline $1.03(3)$ & $3.8755(1)$ & $7.2985(5)$ & $0.1763(2)$ & $0.6422(11)$ & $6.76 \%$ & $4.59 \%$ & 1.794 \\
\hline $2.01(3)$ & $3.8635(1)$ & $7.2531(7)$ & $0.1772(2)$ & $0.6420(8)$ & $5.91 \%$ & $4.41 \%$ & 1.836 \\
\hline $3.10(3)$ & $3.8522(1)$ & $7.1949(7)$ & $0.1775(2)$ & $0.6409(10)$ & $5.02 \%$ & $3.80 \%$ & 1.479 \\
\hline $3.99(5)$ & $3.8420(2)$ & $7.1582(9)$ & $0.1784(3)$ & $0.6402(13)$ & $4.99 \%$ & $3.86 \%$ & 1.678 \\
\hline $5.03(5)$ & $3.8280(2)$ & $7.1213(9)$ & $0.1798(3)$ & $0.6395(18)$ & $4.66 \%$ & $3.75 \%$ & 1.564 \\
\hline $5.98(5)$ & $3.8184(2)$ & 7.0897(9) & $0.1813(2)$ & $0.6373(18)$ & $4.28 \%$ & $3.42 \%$ & 1.403 \\
\hline $7.04(7)$ & $3.8084(2)$ & $7.0578(10)$ & $0.1822(2)$ & $0.6369(17)$ & $4.19 \%$ & $3.32 \%$ & 1.360 \\
\hline $8.06(6)$ & $3.8022(2)$ & $7.0247(10)$ & $0.1826(2)$ & $0.6362(17)$ & $4.19 \%$ & $3.29 \%$ & 1.316 \\
\hline $9.06(7)$ & $3.7942(2)$ & $7.0019(10)$ & $0.1837(2)$ & $0.6359(19)$ & $3.95 \%$ & $3.01 \%$ & 1.211 \\
\hline $10.11(9)$ & $3.7855(2)$ & $6.9819(11)$ & $0.1835(2)$ & $0.6354(20)$ & $3.75 \%$ & $2.99 \%$ & 1.211 \\
\hline $11.08(8)$ & $3.7781(2)$ & $6.9639(12)$ & $0.1838(2)$ & $0.6355(20)$ & $3.58 \%$ & $2.91 \%$ & 1.211 \\
\hline $12.24(9)$ & $3.7686(2)$ & $6.9404(12)$ & $0.1844(2)$ & $0.6354(21)$ & $3.69 \%$ & $2.89 \%$ & 1.242 \\
\hline $13.21(12)$ & $3.7660(2)$ & $6.9215(12)$ & $0.1853(3)$ & $0.6348(21)$ & $3.70 \%$ & $2.98 \%$ & 1.255 \\
\hline $14.22(16)$ & $3.7629(3)$ & $6.9068(13)$ & $0.1861(3)$ & $0.6348(22)$ & $3.71 \%$ & $3.04 \%$ & 1.329 \\
\hline $15.14(9)$ & $3.7636(3)$ & $6.9056(13)$ & $0.1855(3)$ & $0.6344(21)$ & $3.81 \%$ & $3.17 \%$ & 1.411 \\
\hline 16.1(1) & $3.7685(3)$ & $6.8760(13)$ & $0.1861(3)$ & $0.6344(23)$ & $4.06 \%$ & $3.31 \%$ & 1.384 \\
\hline 17.1(1) & $3.7687(3)$ & $6.8391(14)$ & $0.1869(3)$ & $0.6346(24)$ & $4.96 \%$ & $3.85 \%$ & 1.706 \\
\hline $17.9(1)$ & $3.7699(3)$ & $6.7896(18)$ & $0.1870(3)$ & $0.6342(31)$ & $5.43 \%$ & $4.18 \%$ & 1.922 \\
\hline $19.0(1)$ & $3.7711(4)$ & $6.7323(23)$ & $0.1871(4)$ & $0.6344(37)$ & $5.87 \%$ & $4.50 \%$ & 2.333 \\
\hline $20.0(2)$ & $3.7710(4)$ & $6.6999(26)$ & $0.1881(4)$ & $0.6342(40)$ & $6.14 \%$ & $4.81 \%$ & 2.439 \\
\hline $21.1(2)$ & $3.7700(5)$ & $6.6754(31)$ & $0.1886(5)$ & $0.6343(51)$ & $6.77 \%$ & $5.22 \%$ & 2.702 \\
\hline $22.1(2)$ & $3.7683(5)$ & $6.6579(33)$ & $0.1888(5)$ & $0.6351(53)$ & $7.12 \%$ & $5.45 \%$ & 2.852 \\
\hline $23.0(2)$ & $3.7622(5)$ & $6.6384(34)$ & $0.1896(5)$ & $0.6356(60)$ & $7.45 \%$ & $5.56 \%$ & 2.778 \\
\hline $24.5(3)$ & $3.7581(6)$ & $6.6055(38)$ & $0.1894(5)$ & $0.6359(63)$ & $7.63 \%$ & $5.61 \%$ & 2.871 \\
\hline $26.9(3)$ & $3.7493(6)$ & $6.5450(49)$ & $0.1908(7)$ & $0.6360(67)$ & $8.43 \%$ & $6.03 \%$ & 3.280 \\
\hline $28.5(3)$ & $3.7452(6)$ & $6.5191(49)$ & $0.1906(7)$ & $0.6359(71)$ & $8.44 \%$ & $6.17 \%$ & 3.185 \\
\hline $30.1(2)$ & $3.7374(7)$ & $6.5009(49)$ & $0.1917(7)$ & $0.6361(73)$ & $8.35 \%$ & $6.05 \%$ & 3.289 \\
\hline $31.9(3)$ & $3.7321(7)$ & $6.4730(58)$ & $0.1920(9)$ & $0.6360(78)$ & $8.75 \%$ & $6.31 \%$ & 3.563 \\
\hline $34.1(3)$ & $3.7259(7)$ & $6.4370(59)$ & $0.1927(9)$ & $0.6366(80)$ & $8.79 \%$ & $6.34 \%$ & 3.553 \\
\hline
\end{tabular}




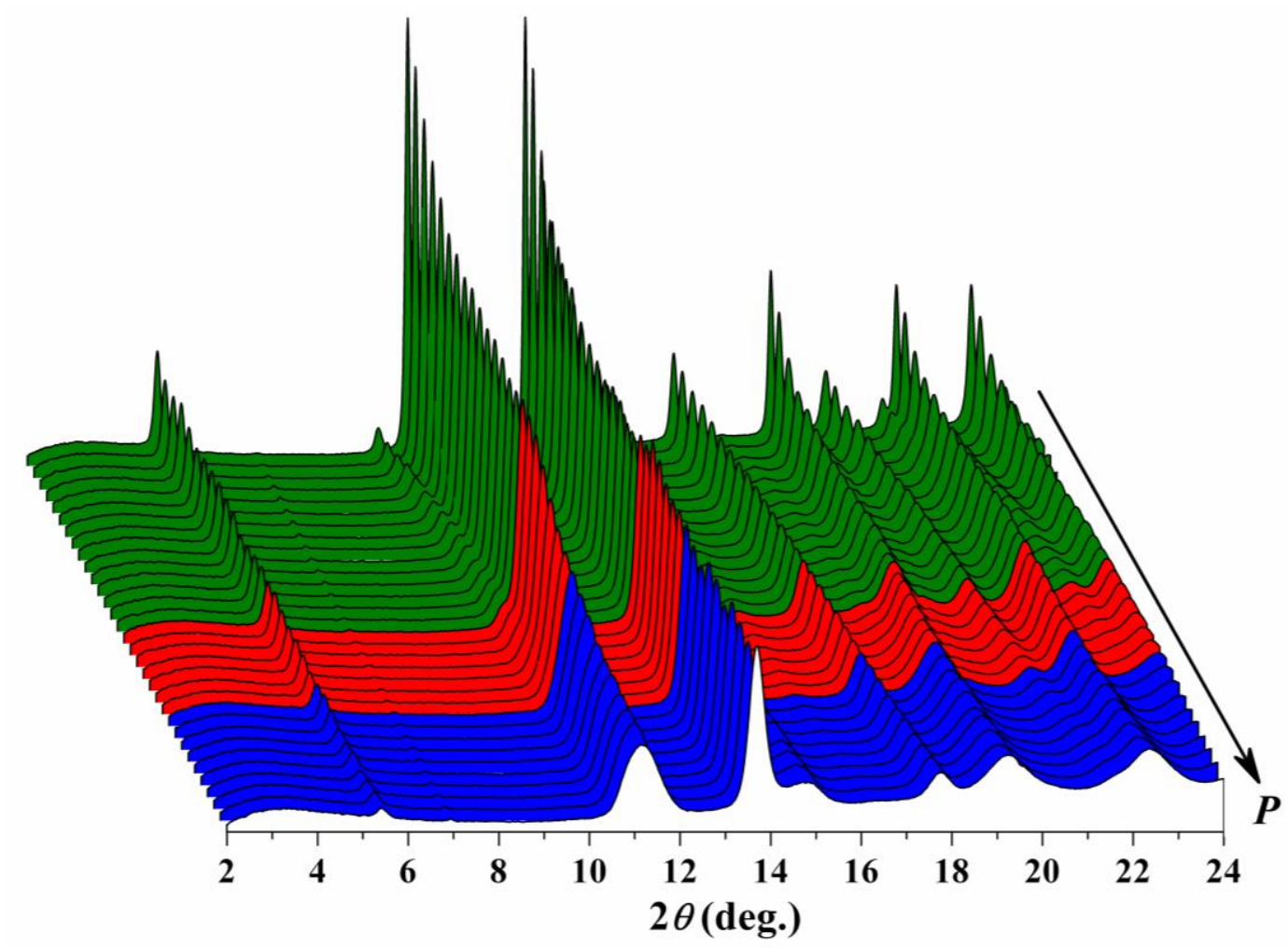

Figure S1. Angle-dispersive X-ray diffraction (XRD) patterns of $\mathrm{BiOCl}$ at room temperature up to $38.9 \mathrm{GPa}(\lambda=0.6199 \AA)$. The green, red, and blue XRD patterns represent the low-pressure tetragonal (LPT) phase, transitional region, and high-pressure tetragonal (HPT) phase, respectively. 


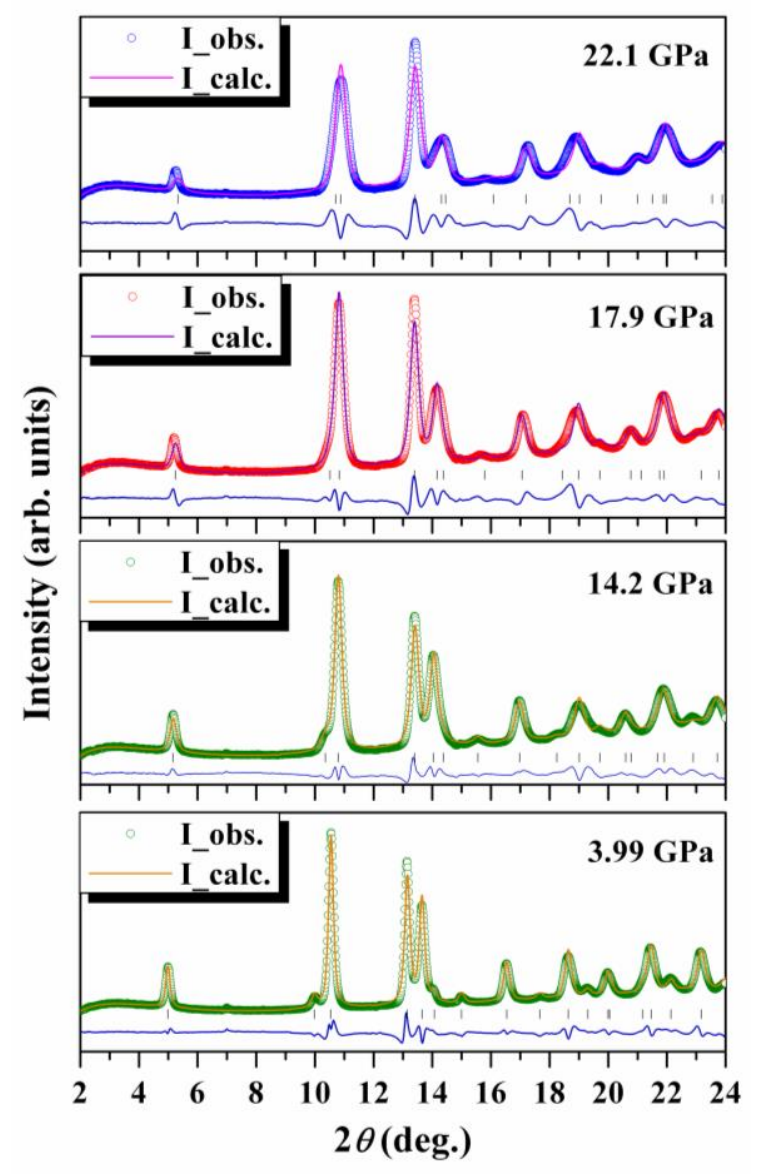

Figure S2. Experimental (open circle) and fitted (line) X-ray diffraction (XRD) patterns for BiOCl at $3.99,14.2,17.9$ and $22.1 \mathrm{GPa}(\lambda=0.6199 \AA)$. The vertical lines are denoted the Bragg peaks. 


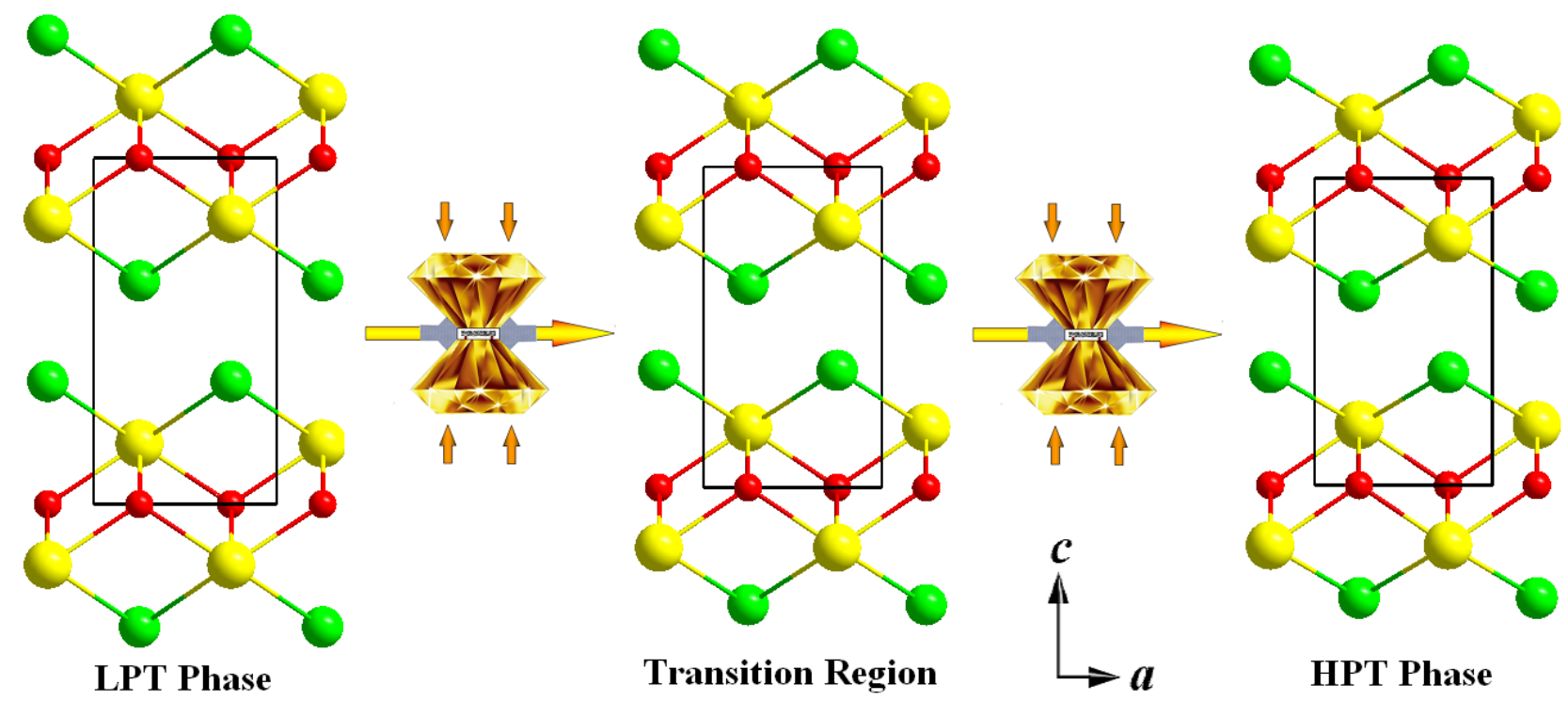

Figure S3. Schematic views of crystal structure evolution under high pressure of BiOCl. The yellow, red, and green globes represent the $\mathrm{Bi}, \mathrm{O}$, and $\mathrm{Cl}$ atoms, respectively. 

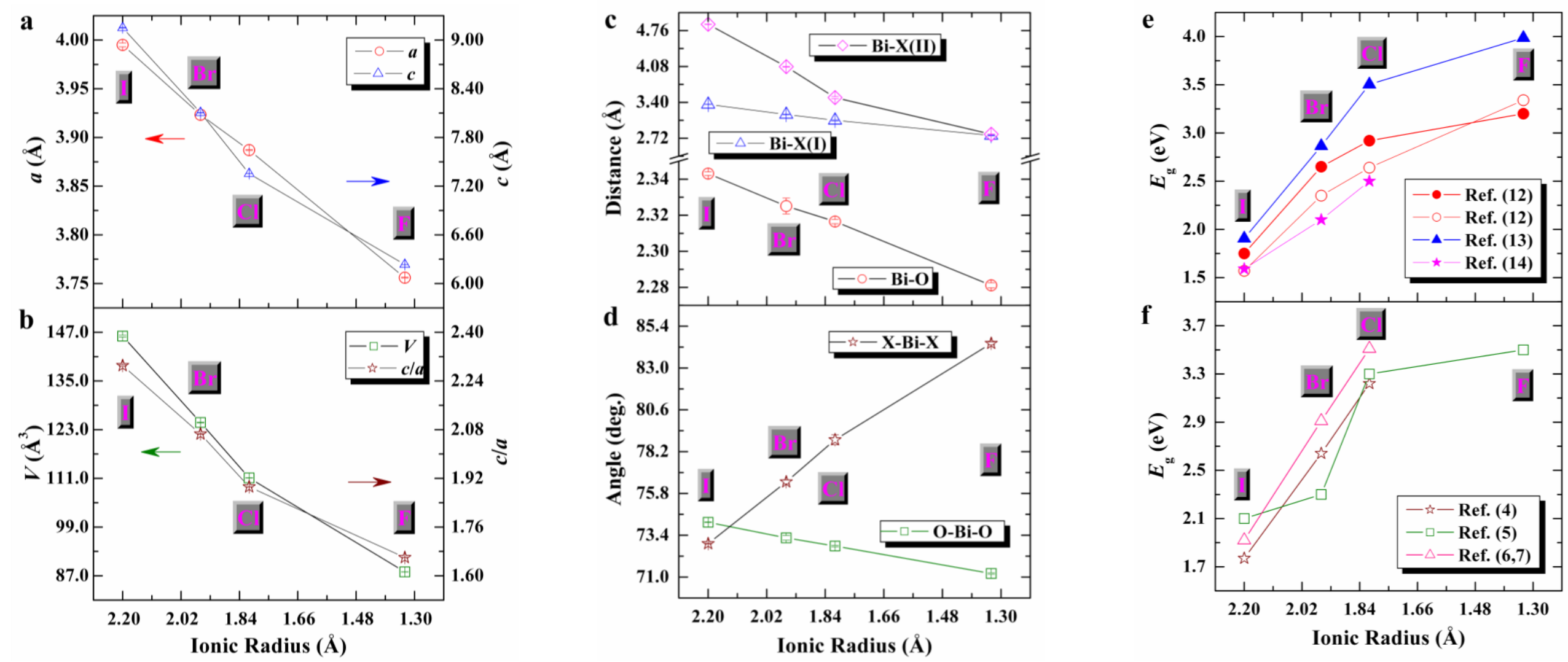

Figure S4. X ionic radius $\left(r_{\mathrm{X}}\right)$ dependences of (a) lattice parameters ( $a$ and $\left.c\right)$, (b) unit cell volume $(V)$ and the $c$ to $a$ ratio $(c / a)$ ratio, (c) bond distances, (d) bond angles, and (e) calculated and (f) experimental band-gap width $\left(E_{\mathrm{g}}\right)$ for $\mathrm{BiOX}\left(\mathrm{X}=\mathrm{F}, \mathrm{Cl}, \mathrm{Br}\right.$, and I). ${ }^{4-7,12-18}$ 

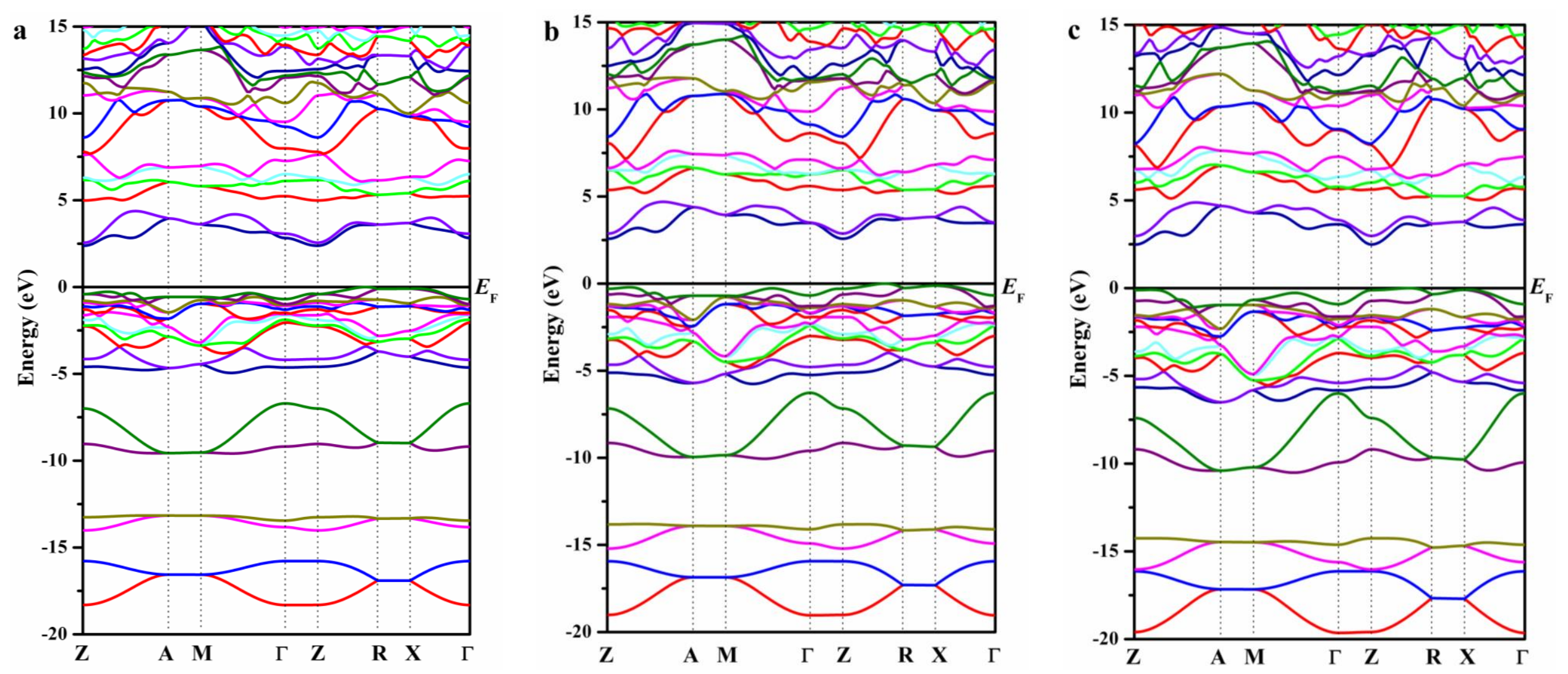

Figure S5. Energy bands of $\mathrm{BiOCl}$ at (a) 0.49, (b) 17.9, and (c) $34.1 \mathrm{GPa}$. The horizontal lines mark the Fermi energy $\left(E_{\mathrm{F}}\right)$. 

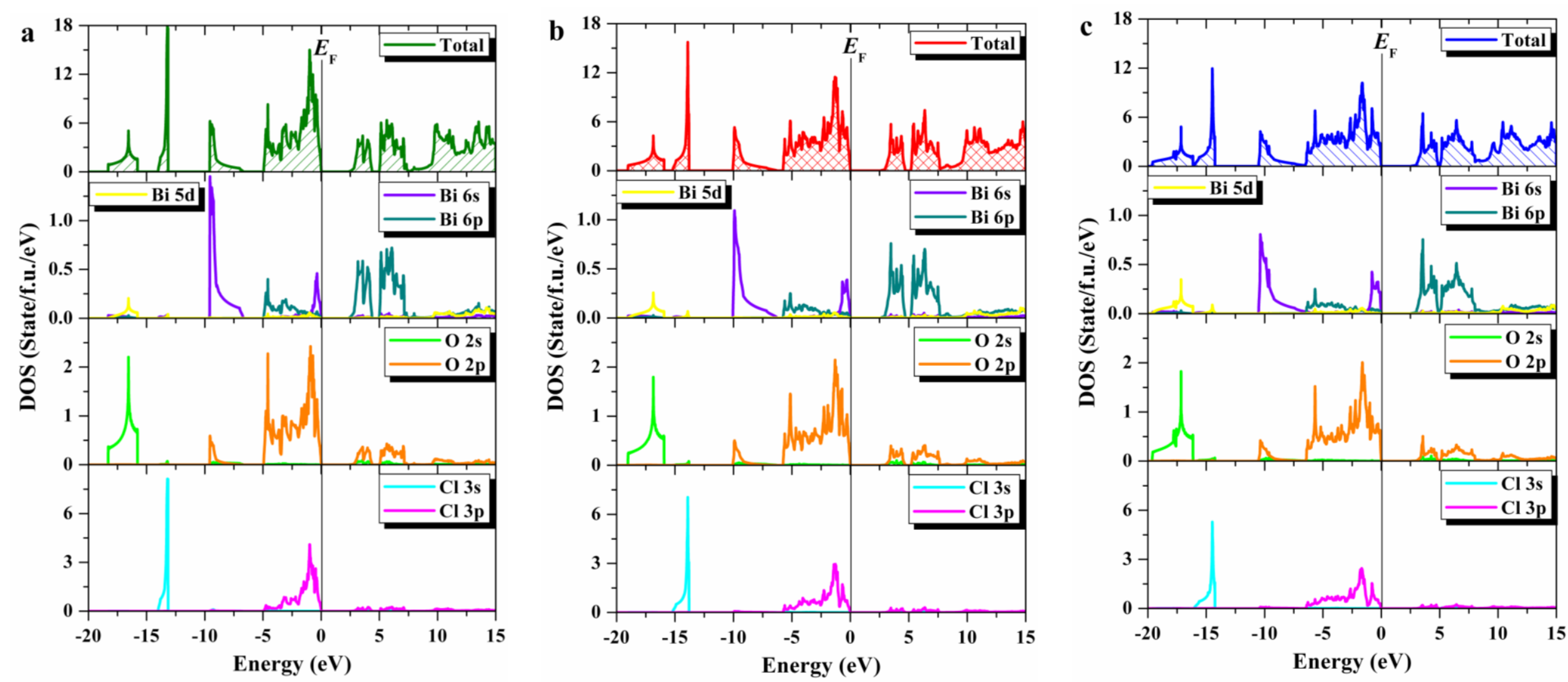

Figure S6. Calculated total and partial density of states (TDOS \& PDOS) of BiOCl at (a) 0.49, (b) 17.9, and (c) 34.1 GPa. The vertical lines mark the Fermi energy $\left(E_{\mathrm{F}}\right)$. 


\section{The complete author list:}

(10) Zhang, K.; Liang, J.; Wang, S.; Liu, J.; Ren, K. X.; Zheng, X.; Luo, H.; Peng, Y. J.; Zou, X.; Bo, X.; Li, J. H.; Yu, X. B. BiOCl Sub-Microcrystals Induced by Citric Acid and Their High Photocatalytic Activities. Cryst. Growth Des. 2011, 12, 793-803.

(24) Ren, Z.-A.; Yang, J.; Lu, W.; Yi, W.; Shen, X.-L.; Li, Z.-C.; Che, G.-C.; Dong, X.-L.; Sun, L.-L.; Zhou, F.; Zhao, Z. X. Superconductivity in Iron-Based F-doped Layered Quaternary Compound $\mathrm{Nd}\left[\mathrm{O}_{1-x} \mathrm{~F}_{x}\right] \mathrm{Fe}$ As. Europhys. Lett. 2008, 82, 57002.

(27) Zhao, J. G.; Wang, L. H.; Dong, D. W.; Liu, Z. G.; Liu, H. Z.; Chen, G. F.; Wu, D.; Luo, J. L.; Wang, N. L.; Yu, Y.; Jin, C. Q.; Guo, Q. Z. Structure Stability and Compressibility of Iron-based Superconductor $\mathrm{Nd}\left(\mathrm{O}_{0.88} \mathrm{~F}_{0.12}\right) \mathrm{Fe} A s$ Under High Pressure. J. Am. Chem. Soc. 2008, 130, $13828-13829$.

(29) Kreyssig, A.; Green, M. A.; Lee, Y.; Samolyuk, G. D.; Zajdel, P.; Lynn, J. W.; Bud'ko, S. L., Torikachvili, M. S.; Ni, N.; Nandi, S.; Leão, J. B.; Poulton, S. J.; Argyriou, D. N.; Harmon, B. N.; McQueeney, R. J.; Canfield, P. C.; Goldman, A. I. Pressure-Induced Volume-Collapsed Tetragonal Phase of $\mathrm{CaFe}_{2} \mathrm{As}_{2}$ as Seen via Neutron Scattering. Phys. Rev. B 2008, 78, 184517.

(30) Mittal, R.; Heid, R.; Bosak, A.; Forrest, T. R.; Chaplot, S. L.; Lamago, D.; Reznik, D.; Bohnen, K.-P.; Su, Y.; Kumar, N.; Dhar, S. K.; Thamizhavel, A.; Rüegg, Ch.; Krisch, M.; McMorrow, D. F.; Brueckel, Th.; Pintschovius, L. Pressure Dependence of Phonon Modes across the Tetragonal to Collapsed-Tetragonal Phase Transition in $\mathrm{CaFe}_{2} \mathrm{As}_{2}$. Phys. Rev. B 2010, 81, 144502.

(32) Mittal, R.; Mishra, S. K.; Chaplot, S. L.; Ovsyannikov, S. V.; Greenberg, E.; Trots, D. M.; Dubrovinsky, L.; Su, Y.; Brueckel, Th.; Matsuishi, S.; Hosono, H.; Garbarino, G. Ambient- and Low-Temperature Synchrotron X-ray Diffraction Study of $\mathrm{BaFe}_{2} \mathrm{As}_{2}$ and $\mathrm{CaFe}_{2} \mathrm{As}_{2}$ at High Pressures up to 56 GPa. Phys. Rev. B 2011, 83, 054503.

(35) Yu, Z. H.; Wang, L.; Wang, L. H.; Liu, H. Z.; Zhao, J. G.; Li, C. Y.; Sinogeikin, S.; Wu, W.; Luo, J. L.; Wang, N. L.; Yang, K.; Zhao, Y. S.; Mao, H.-K. Conventional Empirical Law Reverses in the Phase Transitions of 122-type Iron-Based Superconductors. Sci. Rep. 2014, 4, 7172.

(36) Liu, Q. Q.; Yu, X. H.; Wang, X. C.; Deng, Z.; Lv, Y. X.; Zhu, J. L.; Zhang, S. J.; Liu, H. Z.; Yang, W. G.; Wang, L.; Mao, H. K.; Shen, G. Y.; Lu, Z. Y.; Ren, Y.; Chen, Z. Q.; Lin, Z. J.; Zhao, Y. S.; Jin, C. Q. Pressure-Induced Isostructural Phase Transition and Correlation of FeAs Coordination with the Superconducting Properties of 111-Type $\mathrm{Na}_{1-x} \mathrm{FeAs}$. J. Am. Chem. Soc. 2011, 133, $7892-7896$. 